\title{
COSTO DE CAPITAL PARA EL SECTOR VITIVINÍCOLA CHILENO: UNA PROPUESTA DESDE EL MODELO DE VALORACIÓN DE ACTIVOS DE CAPITAL (CAPM)
}

\section{Cost of capital for the Chilean wine sector: a proposal using the Capital Asset Pricing Model (CAPM)}

\author{
Pablo Morán V. ${ }^{1}$ *
}

\begin{abstract}
R E S U M E N
El costo de capital es un insumo clave para obtener el valor fundamental de un activo o proyecto. En este trabajo se estima el costo de capital del sector vitivinícola chileno a través del Modelo de Valoración de Activos de Capital (CAPM, del inglés Capital Asset Pricing Model). Se discuten brevemente las dificultades que emergen al utilizar este modelo de equilibrio en la práctica, y la literatura empírica reciente sobre la estimación del coeficiente de riesgo sistemático (Beta). Basado en una muestra de empresas del sector vitivinícola chileno, e información histórica y de mercado, se encontró que el costo de capital asociado al giro vitivinícola en Chile asciende a $8,9 \%$ real anual.
\end{abstract}

Palabras clave: tasa de descuento, estimación robusta, ajuste Bayesiano.

\begin{abstract}
A B S T R A C T
The cost of capital is a key input to obtain the fundamental value of an asset or project. In this paper, the cost of capital for the Chilean wine sector was estimated through the Capital Asset Pricing Model (CAPM). We briefly discuss the difficulties that arise when this equilibrium model is used in practice, and the most recent empirical literature dealing with the estimation of the systematic risk coefficient (Beta). From a sample of Chilean wine enterprises, using historical and market information, we found that the cost of capital for the wine sector activities in Chile was of $8.9 \%$ per year in real terms.
\end{abstract}

Key words: discount rate, robust estimation, Bayesian adjustment.

\footnotetext{
${ }^{1}$ Universidad de Talca, Facultad de Ciencias Empresariales, 2 Norte 685, Casilla 721, Talca-Chile.

Email:pmoran@utalca.cl *Autor para correspondencia.

Recibido: 3 de julio de 2006. Aceptado: 12 de septiembre de 2006.
} 


\section{INTRODUCCIÓN}

La tasa de costo de capital (tasa de descuento) es un insumo fundamental en la valoración de un activo mediante flujos de caja descontados. Conceptualmente, ésta representa la tasa de rentabilidad ofrecida por alternativas de inversión de riesgo comparable y, por lo tanto, pasa a ser la rentabilidad a la que se renuncia (costo de oportunidad) por invertir en un activo determinado (Brealey y Myers, 2003).

El Modelo de Valoración de Activos de Capital (CAPM, Capital Asset Pricing Model) ha sido tradicionalmente utilizado para determinar la rentabilidad exigida a una inversión. Aun cuando en la actualidad existe un importante debate académico sobre la validez del CAPM (Fama y French, 2004), este modelo se mantiene aún como una herramienta de amplio uso en el ámbito práctico. Por ejemplo, en un estudio sobre la práctica de las finanzas en las empresas, Brounen et al. (2004) señalan que entre las empresas que estiman el costo de capital, el CAPM puro tiene una tasa de utilización de $73 \%$ en Estados Unidos, $47 \%$ en Reino Unido, $56 \%$ en Holanda, $34 \%$ en Alemania y $45 \%$ en Francia. En Chile, la legislación que regula los servicios básicos establece que para determinar la tasa de descuento en los procesos tarifarios se debe utilizar el CAPM. Específicamente en la agricultura, Lobos y Muñoz (2005) utilizan el CAPM para determinar indicadores de rentabilidad y eficiencia para cultivos de manzana en la Región del Maule. Acuña y Drake (2003) discuten una serie de artículos que utilizan el CAPM en el ámbito forestal.

Reconociendo la importancia que tiene una evaluación formal de las inversiones de capital para una buena asignación de los recursos financieros, este artículo tiene como principal objetivo la estimación de una tasa de costo de capital para el sector vitivinícola chileno. Se utiliza el CAPM como modelo de valoración y se discute en detalle el proceso de implementación del modelo al sector bajo estudio. Sin embargo, la discusión es suficientemente general como para que la metodología sea aplicada a otros sectores de la agricultura.

\section{MATERIALES Y MÉTODOS}

\section{Marco teórico}

La valoración estándar de una inversión o empresa mediante flujos de caja descontados, consiste en determinar el valor presente de la corriente flujos de caja disponible (residual) para todos aquellos agentes que proveen capital financiero (flujo de caja libre). Bajo este enfoque de valoración, la tasa de descuento asociada a los flujos de caja (costo de oportunidad de los fondos) es el costo de capital promedio ponderado (WACC, Weighted Average Cost of Capital), definido como:

$$
W A C C=K_{d}(1-t) L+K_{p}(1-L)
$$

donde $K_{d}$ representa el costo asociado a la deuda (tasa a la cual la empresa puede endeudarse hoy), $K_{p}$ es el costo de capital del patrimonio, y $t$ es la tasa marginal de impuesto de la empresa. En esta formulación, ambas tasas de rentabilidad son ponderadas por la importancia relativa que tiene cada fuente de financiamiento a largo plazo en la empresa. Así, $L=B /(B+P)$ representa la proporción del activo total a precios de mercado -deuda $(B)$ más patrimonio $(P)$ - que es financiado con deuda. Por otro lado, el costo de capital del patrimonio $\left(K_{p}\right)$ viene a compensar al dueño por el riesgo operacional y el riesgo financiero que éste asume con la inversión, siendo éste definido por Copeland et al. (2005) como:

$K_{p}=K_{u}+\left(K_{u}-K_{d}\right)(1-t)(B / P)$

donde $K_{u}$ corresponde al costo de capital-después de impuestos- asociado al giro operacional del negocio, y $B / P$ es la relación entre deuda y patrimonio a largo plazo de la empresa, ambos a precios de mercado.

Un último elemento necesario para completar esta breve discusión sobre el costo de capital se refiere a los determinantes de cada una de las rentabilidades exigidas. El CAPM establece que, en equilibrio, la rentabilidad exigida del activo $i$ corresponde a:

$E\left(R_{i}\right)=K_{i}=r_{f}+\lambda_{m} \beta_{i} \operatorname{con} \lambda_{m}=E\left(R_{m}\right)-r_{f}$

donde $E\left(R_{i}\right)$ es la rentabilidad esperada del activo $i, K_{i}$ es la rentabilidad exigida del activo $i, r_{f}$ representa la tasa de un instrumento libre de riesgo, $\lambda_{m}$ corresponde a la prima por riesgo (porcentual) asociada al portafolio de todos los activos de la economía (portafolio de mercado, $m$ ), y $\beta_{i}$ es el coeficiente de riesgo sistemático (Beta) del activo $i$. Este último coeficiente viene dado por la relación entre la covarianza del retorno del activo $i$ y el 
retorno del portafolio de mercado $(m)$, y la varianza del portafolio de mercado; formalmente:

$$
\beta_{i}=\frac{\sigma_{i, m}}{\sigma_{m}^{2}}
$$

En la ecuación (2), el costo de capital del patrimonio $\left(K_{p}\right)$, el costo de capital asociado al giro operacional $\left(K_{u}\right)$ y el costo asociado a la deuda $\left(K_{d}\right)$ representan tasas exigidas de rentabilidad o tasas de rentabilidades esperadas de equilibrio. Así, en principio, es posible determinar sus magnitudes a través del CAPM. Utilizando el CAPM para representar las tasas de rentabilidad exigida que aparecen en la ecuación (2), es posible demostrar (Copeland et al., 2005) que el coeficiente de riesgo sistemático (Beta) asociado al giro operacional corresponde a:

$$
\beta_{u}=\frac{\beta_{p}+\beta_{d}(B / P)(1-t)}{1+(B / P)(1-t)}
$$

donde $\beta_{p}$ y $\beta_{d}$ representan el riesgo sistemático (Beta) asociado al patrimonio y a la deuda, respectivamente.

Dos observaciones permitirán acotar este trabajo. Primero, considerando que el costo de capital promedio ponderado (WACC) y el costo de capital del patrimonio $\left(K_{p}\right)$ dependen del nivel de deuda objetivo de la empresa ( $L$ o alternativamente $B / P$ ), en este trabajo se presentará una estimación de la tasa de costo de capital asociada al giro operacional $\left(K_{u}\right)$ del sector vitivinícola chileno. Esta tasa de descuento depende de factores industriales y operacionales relativamente transversales a todas las empresas (o proyectos) que participan en un giro operacional determinado. Con una estimación de la tasa $K_{u}$ y la definición del nivel de deuda objetivo de una empresa específica es posible, usando las ecuaciones (1) y (2), determinar la tasa de costo de capital promedio ponderado del proyecto o empresa.

Segundo, según el CAPM el factor que explica las diferencias en rentabilidades exigidas a los activos es el coeficiente Beta. Esto es así ya que, en teoría, tanto la prima por riesgo del portafolio de mercado $\left(\lambda_{m}\right)$ como la tasa del instrumento libre de riesgo $\left(r_{f}\right)$ son transversales a todos los activos de la economía cuando el horizonte de planeación es común. Por lo tanto, el trabajo que sigue se centrará en la estimación del coeficiente Beta $\left(\beta_{u}\right.$, riesgo sistemático) del sector vitivinícola chileno. Estimaciones previas del premio por riesgo del portafolio de mercado $\left(\lambda_{m}\right)$ en Chile y una tasa libre de riesgo $\left(r_{f}\right)$ adecuada al horizonte de planeación permitirán, a través de la ecuación (3) determinar el costo de capital asociado al giro operacional $\left(K_{u}\right)$.

\section{Estimación del coeficiente Beta}

La estimación de Beta parte tradicionalmente del modelo de mercado definido por la siguiente regresión simple (Copeland et al., 2005):

$$
R_{i t}=a_{i}+b_{i} R_{m t}+e_{i t}
$$

donde $R_{i t}$ es el retorno realizado del activo $i$ para el período $t, R_{m t}$ es el retorno realizado de un índice accionario utilizado como proxy del portafolio de mercado en el período $t, \mathrm{y} e_{i t}$ es el error aleatorio para el activo $i$ en el período $t$. Los coeficientes $a_{i} \mathrm{y}$ $b_{i}$ son los coeficientes a estimar, siendo $b_{i}$ el estimador del coeficiente $\beta_{i}$ en la ecuación (4). Ciertamente este enfoque de estimación para Beta requiere disponer de activos con precios de mercado observables, por ejemplo, activos transados en bolsa. Además, en la estimación del coeficiente Beta de la ecuación (6) es necesario definir la frecuencia y horizonte de tiempo de la medición de los retornos, y el método de estimación de los parámetros. A continuación se describe brevemente la evidencia que sustenta el enfoque empírico para la estimación de Beta en este trabajo.

En principio, la definición del intervalo de medición (diarios, semanales, mensuales, etc.) de los retornos a usar en la ecuación (6) no debería afectar el coeficiente Beta estimado. Sin embargo, Scholes y Williams (1977) y Dimson (1979) demostraron que el estimador mínimo cuadrático ordinario (OLS, del inglés Ordinary Least Squares) del parámetro Beta es sesgado e inconsistente para los activos que se transan más o menos frecuentemente que el promedio de los activos del índice accionario utilizado como proxy del portafolio de mercado. Esta asincronía de los retornos genera una correlación distinta de cero entre el término de error y la variable exógena en la ecuación (6), impidiendo de esta manera que el estimador sea consistente. Ambos trabajos proponen estimadores teóricamente consistentes para Beta a través del uso de rezagos y adelantos del índice accionario utilizado. Sin embargo, investigaciones posteriores cuestionan su efectividad como mecanismo de corrección (Bartholdy y Riding, 1994). Beer (1997) argumenta que un aumento del intervalo de medición de los retornos (por ejemplo, pasar de retornos diarios a retor- 
nos mensuales) puede mitigar el problema de retraso en el ajuste de precios y el estimador OLS de Beta puede acercarse al verdadero parámetro. Brailsford y Josev (1997) encontraron evidencia consistente con esta observación. Consecuentemente, en este trabajo se usarán retornos calculados con los precios de cierre mensual para la estimación del coeficiente Beta.

En segundo lugar, la estimación del coeficiente Beta con datos (retornos) históricos supone cierta estabilidad del coeficiente o, alternativamente, estacionariedad en su proceso generador. De otra manera, cualquier estimación basada en datos históricos no sería útil para proyectar el coeficiente Beta a futuro. Ebner y Neumann (2005) encuentran evidencia de inestabilidad del coeficiente Beta estimado para el mercado alemán. Lam (1999) encontró para Hong Kong que Beta no es estacionario para períodos largos, pero que éste sí presenta cierta estabilidad para períodos de hasta cuatro años. Sus resultados serían consistentes con evidencia encontrada en Estados Unidos, donde existiría cierta estabilidad para períodos de cinco años. Groenewold y Fraser (2000) encuentran para Australia, que un Beta estimado con los retornos mensuales de cinco años genera menores errores de estimación fuera de la muestra que las técnicas simples (por ejemplo, rolling regressions) para modelar un coeficiente Beta variable en el tiempo. A la luz de esta evidencia, la estimación del coeficiente de riesgo sistemático se realizará utilizando un período de estimación de cinco años.

Un problema que potencialmente explica el patrón de variación en el tiempo del Beta estimado es el error de muestreo al que está sujeto cualquier estimador estadístico (sesgo de selección). Vasicek (1973) propuso un estimador Bayesiano que corrige la estimación OLS de Beta a través de la incorporación de información a priori sobre distribución del coeficiente. En términos simples, el estimador ajustado se describe como:

$$
\beta_{i}^{V}=\beta_{x}\left(1-x_{i}\right)+b_{i} x_{i}
$$

donde $b_{i}$ es estimador OLS de Beta de la ecuación (6) para el activo $i$, y $\beta_{x}$ es un estimador de la media poblacional de la distribución a priori del estimador de Beta. Para obtener el estimador Bayesiano, ambos estimadores obtenidos en una primera etapa $\left(b_{i} \mathrm{y} \beta_{x}\right)$ son luego ponderados por el coeficiente $x_{i}$, factor que busca dar mayor o menor peso a cada estimador en función de la precisión de cada uno. Dicho factor se construye (Lally, 1998) a partir de la varianza muestral asociada al estimador OLS $\left(S^{2}\left(b_{i}\right)\right)$ en relación a aquella del estimador a priori $\left(\sigma_{x}^{2}\right)$ a través de la siguiente expresión:

$$
x_{i}=\frac{\sigma_{x}^{2}}{\sigma_{x}^{2}+S^{2}\left(b_{i}\right)}
$$

Murray (1995) encontró que la corrección Bayesiana genera Betas más estables para el mercado irlandés. Evidencia reportada por Beer (1997) para el mercado belga y Luoma et al. (1999) para el mercado finlandés, indican que la corrección Bayesiana mejoraría la estimación del coeficiente de riesgo sistemático. Considerando la potencial inestabilidad del Beta estimado debido a sesgo de selección, en la estimación posterior se incorporará la corrección Bayesiana siguiendo las recomendaciones de Lally (1998).

Por último, es sabido en la literatura econométrica que el estimador OLS de la ecuación (6) es sensible a la presencia de observaciones influyentes (Chan y Lakonishok, 1992). Martin y Simin (2003) evaluaron el desempeño de estimadores robustos ante la presencia de observaciones influyentes, en conjunto con la corrección Bayesiana, para el mercado norteamericano en el período 1962-1996; el estimador robusto propuesto por ellos tiene un poder predictivo mayor que el estimador OLS ante la presencia de observaciones influyentes. Gloete et al. (2002) utilizaron estimadores robustos y ajuste Bayesiano en el mercado sudafricano, y encontraron que con datos diarios los estimadores robustos se desempeñan mejor que los OLS. Sin embargo, con datos mensuales los estimadores robustos parecen aportar menos para obtener un mejor estimador de Beta. Se argumenta que con datos mensuales se diluye el efecto de los "outliers" (observaciones que salen de la norma) sobre la estimación.

Dada la escasa evidencia disponible sobre la efectividad de estimadores robustos, en este trabajo se presentará una estimación de Beta a través de un estimador robusto y el estimador clásico OLS. En particular, se utilizará el "MM-estimator" (robusto a "outliers") con errores estándar robustos a heterocedasticidad y autocorrelación propuesto por Croux et al. (2004). En general, un estimador robusto reduce la ponderación (a través de diversos mecanismos de pesos) que tienen sobre el estimador 
los"outliers". En particular, un "MM-estimator" se obtiene en tres etapas, donde se busca minimizar la siguiente función objetivo:

$$
\sum_{i=1}^{n} \rho\left(\frac{e_{i}}{\hat{s}} ; c\right)
$$

donde $\hat{s}$ es un estimador de escala robusto y $\rho(\bullet ; c)$ es una función de pesos convexa ("convex weight function") de los residuos de la regresión $-e_{i}$ en la ecuación (6)- con constante de ajuste $c$ (Anderson y Schumacker, 2003).

\section{Fuentes de información}

La principal fuente de información es Economática (2006). Esta base de datos incorpora un registro histórico de la información contable y de mercado de las empresas listadas en las bolsas latinoamericanas. La selección de las empresas representativas del sector vitivinícola partió de la lista de empresas chilenas clasificadas por Economática en la industria Alimentos \& Bebidas. De éstas, las empresas del sector vitivinícola son: Concha y Toro, Santa Emiliana, San Pedro, Undurraga y Santa Rita. Como criterio de selección, se mantuvieron en la muestra sólo aquellas empresas que al cierre del año 2005 tenían una presencia bursátil de al menos $25 \%$ en la Bolsa de Comercio de Santiago en los últimos cinco años. Al imponer este criterio, las empresas remanentes y que fueron usadas como representativas del sector vitivinícola chileno son San Pedro, Concha y Toro y Santa Rita. Lobos y Viviani (2007) reportan que a marzo de 2006 estas tres viñas representaban un $73 \%$ de las ventas del mercado vitivinícola doméstico.

Para determinar los parámetros a priori $\left(\beta_{x} \mathrm{y} \sigma^{2}{ }_{x}\right.$ en las ecuaciones 7 y 8 , respectivamente) en la aplicación de la corrección Bayesiana, Lally (1998) recomienda utilizar sólo una submuestra del universo de empresas disponibles; idealmente aquellas que puedan ser caracterizadas como pertenecientes a un universo relativamente homogéneo. En este trabajo se consideró relevante como submuestra a todas las empresas pertenecientes a las industrias Alimentos \& Bebidas y Comercio del listado disponible en Economática. Además de imponer el criterio de presencia bursátil descrito arriba, para empresas que tenían más de una serie de acciones, se eliminó la serie con menor presencia bursátil. Se eliminaron además dos empresas porque las series de precios disponibles no cubrían los cinco años requeridos en la estimación. Además de las tres empresas vitivi- nícolas ya seleccionadas, la muestra de empresas seleccionadas para la corrección Bayesiana quedó compuesta por: Andina, Cervezas, Embonor, Iansa, Watts, D\&S, Detroit, Falabella, Fasa, Pizarreño, Soquicom y Zofri.

Como proxy del portafolio de mercado se utilizó el Índice General de Precios Accionarios (IGPA) de la Bolsa de Comercio de Santiago. Para la determinación de los retornos realizados se utilizó el valor de cierre mensual del IGPA y los precios de cierre mensual de las acciones de las empresas en la muestra, corregidos por dividendos en efectivo disponibles en Economática para el período diciembre 1999 - diciembre 2005. En los casos en que la acción no presentaba transacción para un mes particular, se reemplazó el dato faltante por el último precio disponible. Esto dejó 60 retornos mensuales para cada activo.

Al estimar el coeficiente Beta con retornos accionarios, lo que se obtiene es $\beta_{p}$ de la ecuación (5). Es decir, para recapturar el coeficiente de riesgo sistemático del giro operacional $\left(\beta_{u}\right)$ se requiere, para cada empresa, una estimación del beta de la deuda $\left(\beta_{d}\right)$, de la tasa de impuesto $(t)$ y del nivel de deuda a largo plazo de la empresa $(B / P)$ a precios de mercado. A continuación definimos los criterios de selección y las fuentes de información para estos parámetros de la ecuación (5).

Para obtener el índice Deuda/Patrimonio $(B / P)$, se obtuvo de Economática el indicador Deuda Total Neta/Patrimonio Total Neto de cada empresa para el cierre de cada año en el período 2001-2005 (cinco años). La Deuda Total Neta corresponde a la deuda financiera de corto y largo plazo menos el efectivo, depósitos a plazo e inversiones de corto plazo. El Patrimonio Total Neto excluye el Interés Minoritario. Dado que estos indicadores están a su valor en libros, el indicador Deuda Total Neta/Patrimonio Total Neto se multiplicó año a año por la razón Valor Libro/Valor Bursátil de la acción al cierre de año (disponible en Economática). De esta manera se transformó el patrimonio desde su valor en libros a su precio de mercado (valor bursátil) al cierre de cada año. Se asumió que el valor en libros de la deuda es una buena aproximación de su precio de mercado. La relación $B / P$ utilizada en la ecuación (5) para cada empresa correspondió al promedio de los cinco años del indicador Deuda Total Neta/ Patrimonio Total Neto corregido. 
Para obtener el coeficiente Beta asociado a la deuda de cada empresa, se asumió que el CAPM describe correctamente la prima por riesgo asociada a los instrumentos de deuda emitidos por las empresas y transados en el mercado secundario local. Así, según la ecuación (3), el coeficiente Beta de la deuda correspondería a la razón entre la prima por riesgo de la deuda de la empresa $\left(K_{d}-r_{f}\right)$ y el premio por riesgo del portafolio de mercado $\left(\lambda_{m}\right)$. Para obtener la prima por riesgo de la deuda, se extrajo de RiskAmerica (2006) el indicador "TIR Spread" calculado para la deuda emitida por las tres empresas vitivinícolas de la muestra. Este "spread" se calcula como el diferencial entre el rendimiento a la madurez (TIR) de la deuda de la empresa y el rendimiento a la madurez de un bono sintético con la misma estructura de flujos y con precio calculado con la curva cero de los instrumentos del Banco Central de Chile. Según el registro disponible en RiskAmerica, la deuda emitida por Concha y Toro y Santa Rita tenía al cierre del año 2005 una clasificación de riesgo AA- y un "TIR spread" promedio para las transacciones del año 2005 de 1,13\% real anual. Por otro lado, la deuda de San Pedro aparecía bajo una clasificación de riesgo $\mathrm{A}+\mathrm{y}$ con una "spread" promedio para el año 2005 de 1,32\% real anual. Al dividir este "spread" por la prima por riesgo del mercado se obtuvo el beta de la deuda de cada empresa que se usará en la ecuación (5).

La tasa de impuesto de primera categoría en Chile actualmente es $17 \%$. La práctica común es, siguiendo la literatura de Estados Unidos, utilizar esta tasa en la ecuación (5). Sin embargo, el hecho que en Chile, a diferencia de Estados Unidos, el impuesto pagado en primera categoría (empresa) sirva como crédito a nivel personal al momento del reparto de las utilidades de la empresa hace que el beneficio tributario del uso de deuda sea menor e incluso inexistente. Sólo en el caso en que la empresa retenga sus utilidades (no pago dividendos) en forma indefinida, el beneficio tributario del uso de deuda sería de $17 \%$ por cada peso de gastos financieros (Maquieira y Niño, 1994). En este trabajo se utilizará una tasa marginal de impuestos de $10 \%$ para todas las empresas de la muestra. La tasa elegida está más cerca del $17 \%$ porque según la información de Economática para los últimos tres años, la relación entre el dividendo y la utilidad por acción ("payout ratio") de las empresas de la muestra sobrepasa levemente el $40 \%$. Es decir, es algo mayor que el mínimo de $30 \%$ exigido a las socieda- des anónimas abiertas, pero está lejos del 100\% que resultaría al repartir todas las utilidades del período. En todo caso, dado los niveles de deuda de las empresas vitivinícolas, la elección de esta tasa no tiene un efecto significativo en la estimación del costo de capital.

Como paso final, se necesita una estimación de la tasa del instrumento libre de riesgo $\left(r_{f}\right)$ y de la prima por riesgo asociada al portafolio de mercado $\left(\lambda_{m}\right)$. En relación a la primera variable, es importante que el instrumento elegido esté libre de riesgo de no pago, riesgo de inflación y riesgo de refinanciamiento. Las primeras dos características se cumplen para instrumentos emitidos en unidad de fomento (UF) por el Banco Central de Chile (bonos BCU) y la Tesorería General de la República (bonos BTU). El tercer requisito implica que debe haber un calce (matching) entre el horizonte de planeación de la inversión y la madurez del instrumento a utilizar. Aun cuando este calce es teóricamente recomendado, al momento de escribir este artículo la estructura temporal de las tasas de interés se mostraba bastante plana. Por ejemplo, el rendimiento a la madurez (TIR) promedio para las transacciones registradas en RiskAmerica durante diciembre de 2005 de los BCU fue 3,22\% real anual para instrumentos con madurez entre 4 y 6 años. Por otro lado, el rendimiento a la madurez en el mismo período para las transacciones de los BTU con madurez entre 14 y 16 años fue 3,51\%. Considerando que la diferencia es sólo 29 puntos base, en la estimación del costo de capital se utilizará como tasa libre de riesgo el promedio que corresponde a $3,4 \%$ real anual.

La magnitud de la prima por riesgo asociada al portafolio de mercado $\left(\lambda_{m}\right)$ es un tema que ha generado bastante controversia entre especialistas en Chile y en el extranjero. En particular en Chile, los procesos recientes -años 2003 y 2004- de regulación tarifaria dejaron ver una diversidad de metodologías y estimaciones del premio por riesgo local. Una de las cosas en las que sí parece haber acuerdo es que el premio por riesgo calculado con datos históricos no provee estimaciones confiables. Por ejemplo, el estudio presentado por las empresas de telefonía móvil -disponible en Subtel (2006)- propuso $8,81 \%$ real anual (en relación a papeles de largo plazo). El estudio de la empresa Telefónica de Coyhaique y Telefónica del Sur propuso 7,85\% real anual, mientras que el estudio de CMET propuso 
$8,48 \%$ real anual. Por otro lado, el regulador cuestionó estas magnitudes y propuso una prima por riesgo de 6,24\% real en relación a papeles de 10 años. En general las resoluciones de las comisiones periciales estuvieron más cerca de la propuesta del regulador con rangos entre 6 y 7\% real anual para esta variable. Basados en esta información, para la estimación del costo de capital se utilizará un premio por riesgo para el portafolio de mercado de $6,5 \%$ real anual.

\section{RESULTADOS Y DISCUSIÓN}

\section{Estimación del coeficiente Beta}

El Cuadro 1 presenta las estimaciones de Beta para las acciones-ecuación (6)-de las tres empresas del sector vitivinícola chileno de la muestra, contrastando el estimador clásico OLS con el estimador robusto a "outliers". En ambos casos se utilizan errores estándar corregidos por heterocedasticidad y autocorrelación. Según el estadístico t-Student, todos los coeficientes son estadísticamente significativos al $1 \%$.

En el Cuadro 1 es posible ver que en los casos estudiados, el estimador robusto no parece proveer estimaciones sustancialmente distintas del estimador OLS. Por ejemplo, si consideramos una prima por riesgo para el portafolio de mercado de $6,5 \%$, la máxima diferencia de rentabilidad exigida produci- da, según la ecuación (6), con uno u otro estimador corresponde a San Pedro con un 0,33\% real anual. El uso de retornos mensuales es potencialmente la explicación del escaso efecto que tienen los "outliers" en la estimación del coeficiente Beta (Gloete et al., 2002). Alternativamente, puede ser el caso que, en el período bajo estudio, las acciones de las empresas no hayan tenido retornos extremos. Estos resultados ciertamente no permiten inferir que, en general, los estimadores robustos no proveen mejora sobre el estimador OLS. Martin y Simin (2003) sugieren realizar el contraste entre los estimadores robustos y los OLS con el fin de establecer potenciales sesgos en el estimador OLS. Argumentan que si el estimador OLS y robusto no difieren en forma sustancial, es preferible utilizar el estimador OLS dadas sus propiedades como estimador. Dada la evidencia presentada, en lo que sigue se consideran sólo los estimadores OLS del Cuadro 1.

El Cuadro 2 muestra los resultados del ajuste Bayesiano propuesto por Vasicek (1973) e implementado según las recomendaciones de Lally (1998). La estimación de los coeficientes a priori se realizó a partir de coeficientes estimados con OLS para las empresas de los sectores Comercio y Alimentos \& Bebidas disponibles en Economática. Los estimadores Bayesianos se obtuvieron a partir de las ecuaciones (7) y (8) y los datos presentados en el Cuadro 2.

Cuadro 1. Resultados de la estimación de coeficientes Beta bajo mínimos cuadrados ordinarios y estimación robusta. Table 1. Results for the estimation of Beta coefficients under ordinary least squares and robust estimation.

\begin{tabular}{lccccc}
\hline $\begin{array}{l}\text { Resultados con } \\
\text { empresa proxy }\end{array}$ & \multicolumn{2}{c}{ Estimación OLS } & & \multicolumn{2}{c}{ Estimación robusta } \\
\cline { 2 - 3 } & Coeficiente $\left(\boldsymbol{b}_{\boldsymbol{i}}\right)$ & t-Student & & Coeficiente $\left(\boldsymbol{b}_{\boldsymbol{i}}\right)$ & t-Student \\
\hline Concha y Toro & 1,11 & 4,83 & & 1,08 & 4,05 \\
San Pedro & 0,72 & 3,01 & & 0,77 & 4,03 \\
Santa Rita & 0,74 & 2,90 & & 0,70 & 2,88 \\
\hline
\end{tabular}

OLS: Ordinary least squares (mínimos cuadrados ordinarios).

Cuadro 2. Resultados del ajuste Bayesiano para los coeficientes Beta de la muestra. Table 2. Results for the Bayesian adjustment of the sample Beta coefficients.

\begin{tabular}{|c|c|c|c|c|c|c|}
\hline & \multicolumn{2}{|c|}{ Coeficientes a priori } & \multicolumn{4}{|c|}{ Coeficientes individuales } \\
\hline & $\begin{array}{c}\text { Media } \\
\left(\beta_{x}\right)\end{array}$ & $\begin{array}{c}\text { Varianza } \\
\left(\sigma_{x}^{2}\right)\end{array}$ & $\begin{array}{c}\text { Beta }_{\mathrm{OLS}} \\
\left(\boldsymbol{b}_{i}\right)\end{array}$ & $\begin{array}{c}\text { Varianza } \\
\left(S^{2}\left(b_{i}\right)\right)\end{array}$ & $\begin{array}{c}\text { Ponderación } \\
\left(x_{i}\right)\end{array}$ & $\begin{array}{c}\text { Beta } \\
\text { Bayesiano }\end{array}$ \\
\hline Concha y Toro & & & 1,11 & 0,053 & 0,65 & 1,08 \\
\hline San Pedro & 1,03 & 0,098 & 0,72 & 0,057 & 0,63 & 0,83 \\
\hline Santa Rita & & & 0,74 & 0,064 & 0,61 & 0,85 \\
\hline
\end{tabular}

OLS: Ordinary least squares (mínimos cuadrados ordinarios). 
En relación al efecto de la corrección Bayesiana, vemos que ésta aproxima el Beta estimado a 1 , valor que por definición, corresponde al coeficiente de riesgo sistemático del portafolio de mercado. La razón es que la media a priori del estimador utilizada corresponde a 1,03 . Los pesos relativos $\left(x_{i}\right.$ en ecuación 7) para el estimador OLS resultaron: Concha y Toro 0,65; San Pedro 0,63; y Santa Rita 0,61. Estos resultados son consistentes con el ajuste $\mathrm{ad}$ hoc realizado en la práctica -independiente de la empresa o sector en cuestión-al Beta estimado con OLS, donde se asume una media $\left(\beta_{x}\right)$ igual a 1 para la distribución a priori del estimador y $0,67 \mathrm{como}$ el peso relativo $\left(x_{i}\right)$ del estimador OLS. Sin embargo, estos resultados no permiten inferir que, en general, la implementación adecuada de la corrección Bayesiana no tiene una retribución importante en términos de una mejor estimación del coeficiente Beta.

\section{Costo de capital asociado al giro vitivinícola $\left(K_{u}\right)$} En el Cuadro 3 se presenta el coeficiente Beta asociado al giro operacional del sector vitivinícola $\left(\beta_{u}\right)$ de las tres empresas vitivinícolas de la muestra, determinado a través de la ecuación (5). Se presenta la estimación del Beta asociado a la deuda, obtenido al dividir el "TIR-Spread" de la deuda de cada empresa por la prima por riesgo del mercado $(6,5 \%)$. La relación deuda a patrimonio $(B / P)$ corresponde al promedio de cinco años de la razón Deuda Neta a Patrimonio Neto corregida por la relación Libro/Bolsa de la acción de la empresa. Se considera una tasa de impuesto marginal de 10\% para las tres empresas. En todos los casos se parte del estimador OLS con ajuste Bayesiano (última columna en Cuadro 2), para luego, a partir de las variables descritas en el Cuadro (3), obtener el coeficiente Beta (desapalancado) asociado al giro operacional $\left(\beta_{u}\right)$ promedio de las tres empresas.

Es importante mencionar que los coeficientes Beta estimados para la deuda son consistentes con el nivel de deuda (corregido) utilizado por las empresas. Es decir, las empresas que presentan menores niveles de deuda, presentan también un menor Beta asociado a la deuda (menor riesgo). Por otro lado, el bajo endeudamiento de las empresas de la muestra lleva a que el ajuste por riesgo financiero (desapalancamiento) del Beta accionario no tenga efectos importantes. Por ejemplo, y considerando una prima por riesgo para el portafolio de mercado de $6,5 \%$, la prima por riesgo financiero más alta está asociada a San Pedro con un $0,53 \%$ real anual.

En teoría, el coeficiente Beta asociado al giro operacional $\left(\beta_{u}\right)$ debería ser el mismo para empresas que participan en un mismo giro operacional y que tengan una estructura de activos similar (Mandelker y Rhee, 1984). Aun cuando esta situación no parece reflejarse en el Cuadro 3 para los estimadores muestrales, no podemos descartar que dichas diferencias se deban a la varianza asociada al estimador muestral. Desafortunadamente cualquier prueba de hipótesis formal de esta proposición es cuestionable debido a la serie de ajustes que se han hecho sobre el estimador OLS original $\left(b_{i}\right.$ en el Cuadro 1) y sobre su distribución muestral. En este sentido, se asume -basado en lo que la teoría establece- que los tres estimadores puntuales del Beta asociado al giro operacional en el Cuadro 3 han sido extraídos de una misma población. Por lo tanto, el promedio simple de tres estimadores puntuales debería reflejar una estimación insesgada del parámetro poblacional de interés. Dicho promedio corresponde a $\beta_{u}=0,85$ en el Cuadro 3. En base a esta estimación, un premio por riesgo del portafolio de mercado de $6,5 \%$ y una tasa libre de riesgo de $3,4 \%$, permite establecer a través de la ecuación 3 que la tasa de costo de capital asociada al giro operacional del sector vitivinícola chileno asciende a $8,9 \%$ real anual.

\section{Cuadro 3. Determinación del coeficiente Beta asociado al giro operacional Table 3. Determination of the Beta coefficient for operating activities}

\begin{tabular}{lcccc}
\hline & Beta $_{\text {acción }}\left(\beta_{p}{ }^{p}\right)$ & Beta $_{\text {deuda }}\left(\beta_{d}\right)$ & $\boldsymbol{B} / \boldsymbol{P}(\mathbf{\%})$ & Beta $_{\text {giro }}\left(\beta_{u}\right)$ \\
\hline Concha y Toro & 1,08 & 0,17 & 11,20 & 0,999 \\
San Pedro & 0,83 & 0,20 & 17,05 & 0,748 \\
Santa Rita & 0,85 & 0,17 & 10,83 & 0,791 \\
Beta promedio & - & - & - & 0,846 \\
\hline
\end{tabular}

Los valores finales se calcularon con todos los decimales.

B/P: relación Deuda:Patrimonio ajustada por la relación Libro:Bolsa. 


\section{Consideraciones finales}

La tasa de costo de capital propuesta en este artículo puede ser utilizada, en conjunto con las ecuaciones 1 y 2 , para determinar el costo de capital promedio ponderado (WACC) asociado a las inversiones en el sector vitivinícola. Es importante tener presente que la tasa de costo de capital propuesta está expresada en términos reales (netos de inflación). Por lo tanto, los flujos de caja a descontar deberían también estar expresados en términos reales (en UF por ejemplo) para mantener la consistencia. Aun cuando la presentación de la tasa de costo de capital asociada al giro operacional cumple con el objetivo establecido en este trabajo, se cree importante mencionar algunas limitaciones que permitan al lector ponderar las ventajas y desventajas de utilizar la estimación propuesta.

En primer término, se debe establecer que la prima por riesgo asociada al portafolio de mercado y la tasa de interés libre de riesgo que se utilizaron en la estimación pueden cambiar a futuro. En este sentido, sería recomendable recalcular el costo de capital asociado al giro operacional con el Beta presentado en el Cuadro 3 y con los parámetros que prevalezcan al momento de su uso. Por ejemplo, en base a información de mercado al cierre del año 2005 , se propuso utilizar una tasa de interés libre de riesgo de 3,4\%. Ciertamente esta tasa puede variar a futuro, y puede ser distinta para distintos horizontes de inversión (ver la discusión previa). Lo mismo es relevante para la prima por riesgo asociada al portafolio de mercado.

En segundo lugar, la estimación propuesta descansa fuertemente en la validez del CAPM. La simplicidad y elegancia de este modelo teórico de valoración lo mantienen hasta hoy como uno de los pilares fundamentales en finanzas. Es importante precisar, sin embargo, que las pruebas empíricas del modelo han revelado una serie de anomalías que el CAPM no es capaz de explicar. Por ejemplo, las empresas pequeñas parecen tener un retorno promedio histórico más alto que lo que el CAPM predice. Fama y French (2004) discuten los principales problemas que, según ellos, invalidarían el CAPM, al menos en la manera en que este modelo se utiliza en la práctica. Desafortunadamente, el CAPM como tal es un modelo que no puede ser validado empíricamente dada la imposibilidad de observar el portafolio de mercado (Roll, 1977). Por otro lado, los modelos alternativos como el APT (del inglés Arbi- trage Pricing Theory) y el I-CAPM (Intertemporal CAPM) presentan importantes desafíos al momento de implementarlos en la práctica. Dado esto, no es extraño que Brounen et al. (2004) hayan encontrado que el CAPM es uno de los modelos más utilizados por las grandes empresas norteamericanas y europeas para la estimación de tasas de descuento. Evidencia exploratoria reportada por Moraleda y Quezada (2006), en base al cuestionario y metodología utilizados por Graham y Harvey (2001) para los Estados Unidos, indica que la popularidad del CAPM también es un fenómeno compartido por las grandes empresas chilenas transadas en la bolsa.

Finalmente, el coeficiente Beta presentado en el Cuadro 3 fue estimado a partir de los retornos accionarios de empresas relativamente grandes y transadas en la bolsa. Además, al utilizar precios accionarios observados en el mercado secundario, los precios reflejan el valor que los inversionistas asignan a una participación minoritaria (no controladora) en la empresa. Así, la tasa de costo de capital propuesta refleja exclusivamente la compensación por riesgo sistemático, es decir, asume que el riesgo no sistemático de la empresa o proyecto puede eliminarse al mantener una cartera de inversiones bien diversificada localmente. Aun cuando esto es consistente con lo que la teoría financiera sugiere, Pereiro (2001), entre otros, argumenta que la posibilidad de diversificar el riesgo no sistemático es la excepción y no la regla. En base a una muestra de 91 transacciones de compra y venta de empresas argentinas en el período 1990-1999, Pereiro (2001) sugiere ajustes multiplicativos al valor presente de los flujos de caja de las siguientes magnitudes: tamaño $51 \%$, control 39\% y liquidez 35\%.

Menor acceso a crédito y vulnerabilidad ante bajas en la actividad económica son factores que podrían justificar una tasa de descuento mayor (o un menor valor presente de los flujos de caja) para empresas pequeñas. Otro elemento que induciría a los inversionistas a exigir una mayor tasa de descuento es un premio por la liquidez que conlleva la inversión en una empresa que no transa en la bolsa. Finalmente, la posibilidad de controlar los destinos de la empresa, harían que el control (participación mayoritaria) tenga un valor adicional (menor tasa de descuento) que no es capturado por quienes tienen una participación minoritaria. Ciertamente estas estimaciones de los premios asociados a estos factores no sistemáticos pueden no corresponder al caso chileno. 
Sin embargo, no hay estimaciones equivalentes disponibles en Chile. Dado el orden de magnitud de estos ajustes para el caso argentino, una estimación para el caso chileno es un camino obligado en la agenda futura de investigación.

\section{CONCLUSIONES}

Una breve revisión de los estudios previos sobre estimación del riesgo sistemático (Beta) no permite rechazar la práctica común de estimar este coeficiente con los retornos accionarios mensuales de los últimos cinco años. Al menos para el sector analizado en este estudio, la implementación de una técnica de estimación robusta a "outliers" no parece proveer una estimación sustancialmente distinta a la obtenida con estimadores clásicos OLS. Por otro lado, la aplicación del ajuste Bayesiano entrega estimaciones del coeficiente Beta que no distan de la cruda corrección (comúnmente utilizada) que asume un valor 1 para la media a priori de la distribución del estimador y 0,67 para el ponderador del estimador OLS. Esto lleva a pensar que las dificultades asociadas a la implementación de técnicas complejas de corrección y estimación del coeficiente Beta podrían no retribuir en términos de una mejor estimación. Si bien es cierto estos resultados son consistentes con esta hipótesis, este estudio no puede ser tomado como evidencia a favor o en contra de esta hipótesis, debido a la metodología y foco acotado del estudio. Sin embargo, los resultados son sugerentes, y ciertamente demandan una exploración más profunda del tema en investigaciones futuras.

Los resultados obtenidos a partir de una muestra de empresas del sector vitivinícola indican que la tasa de costo de capital asociada al giro operacional sería levemente inferior al 9\% real anual. Esta tasa se obtuvo a través del CAPM, utilizando el coeficiente Beta desapalancado promedio de tres empresas del sector, una tasa libre de riesgo de 3,4\% y una prima por riesgo para el mercado de $6,5 \%$. Las empresas de la muestra no parecen hacer uso de la deuda en forma intensiva. Esto se refleja en las buenas clasificaciones de riesgo y el bajo premio por riesgo de la deuda emitida por estas empresas. Dado esto, es posible inferir que la tasa de costo de capital promedio ponderado ( WACC) no debería ser en general mucho más baja que la tasa de costo de capital propuesta en este artículo para el giro operacional. Por ejemplo, si se toman los datos promedio de la muestra utilizada, se obtiene que el costo de capital promedio ponderado estaría en torno al 8,8\% real anual. Así, y aun cuando teóricamente los flujos de caja libres deben ser descontados al costo de capital promedio ponderado, el uso de la tasa de rentabilidad exigida propuesta para el giro operacional en el descuento de flujos de caja libres en la práctica no representaría una sobreestimación seria de la tasa de costo de capital correcta.

\section{RECONOCIMIENTOS}

Se agradece a Gonzalo Cortázar y a FINlabUC (Laboratorio de Investigación Avanzada en Finanzas) de la Pontificia Universidad Católica de Chile por proveer acceso a RiskAmerica. Los comentarios de los árbitros anónimos y del editor ayudaron a mejorar sustancialmente este artículo. Cualquier potencial error u omisión es de exclusiva responsabilidad del autor. 


\section{LITERATURA CITADA}

Acuña, E., y F. Drake. 2003. Análisis del riesgo en la gestión forestal e inversiones silviculturales: una revisión bibliográfica. Bosque (Valdivia) 24:113-124.

Anderson, C., and R. Schumacker. 2003. A comparison of five robust regression methods with ordinary least squares regressions: relative efficiency, bias, and test of the null hypothesis. Understanding Statistics 2:79103.

Bartholdy, J., and A. Riding. 1994. Thin trading and the estimation of betas: the efficacy of alternative techniques. J. Finan. Res. 17:241-254.

Beer, F. 1997. Estimation of risk on the Brussels Stock Exchange: methodological issues and empirical results. Global Finance J. 8:83-94.

Brailsford, T., and T. Josev. 1997. The impact of the return interval on the estimation of systematic risk. PacificBasin Finance J. 5:357-376.

Brealey, R., y S. Myers. 2003. Principios de finanzas corporativas. 765 p. Editorial McGraw-Hill, Madrid, España.

Brounen, D., A. de Jong, and K. Koedijk. 2004. Corporate finance in Europe: confronting theory with practice. Finan. Manage. 33:71-101.

Chan, L., and J. Lakonishok. 1992. Robust measurement of beta risk. J. Finan. Quant. Anal. 27:265-282.

Copeland, T., J. Weston, and K. Shastri. 2005. Financial theory and corporate policy. 1000 p. Editorial Pearson Addison Wesley, New York, USA.

Croux, C., G. Dhaene, and D. Hoorelbeke. 2004. Robust standard errors for robust estimators. Department of Applied Economics, K.U. Leuven. Available at http:/ /www.econ.kuleuven.be/ew/academic/econmetr/ members/Dhaene/papers/rsejan2004.pdf Accessed January 2006.

Dimson, E. 1979. Risk measurement when shares are subject to infrequent trading. J. Finan. Econ. 7:197226.

Ebner, M., and T. Neumann. 2005. Time-varying betas of German Stock Returns. Finan. Markets Portfolio Manage. 19:29-46.

Economática. 2006. Tools for investment analysis. Available at http://www.economatica.com Accessed January 2006.

Fama, E., and K. French. 2004. The capital asset pricing model: theory and evidence. J. Econ. Perspect. 18:2546.

Gloete, G., P. de Jongh, and T. de Wet. 2002. Combining Vasicek and robust estimators for forecasting systematic risk. Invest. Analyst J. 55:37-44.

Graham, J., C. Harvey. 2001. The theory and practice of corporate finance: evidence from the field. J. Finan. Econ. 60:187-243.
Groenewold, N., and P. Fraser. 2000. Forecasting beta: how well does the 'five-year rule of thumb' do? J. Bus. Finance Acc. 27:953-982.

Lally, M. 1998. An examination of Blume and Vasicek betas. Finan. Rev. 33:183-198.

Lam, K. 1999. Some evidence on the distribution of beta in Hong Kong. Appl. Finan. Econ. 9:251-262.

Lobos, G., y T. Muñoz. 2005. Indicadores de rentabilidad y eficiencia económica de la producción de manzanas cv. Gala en la Región del Maule, Chile. Agric. Téc. (Chile) 65:421-436.

Lobos, G., et J.L. Viviani. 2007. Estimation des indicateurs de concentration et d'instabilite dans I'industrie vitivinicole du Chili, période 2001-2006. Agroalim. 24:55-61.

Luoma, M., T. Martikainen, and J. Perttunen. 1999. A pseudo criterion for security betas in the Finnish stock market. Appl. Econ. 28:65-69.

Mandelker, G., and G. Rhee. 1984. The impact of the degrees of operating and financial leverage on systematic risk of common stocks. J. Finan. Quant. Anal. 19:45-57.

Maquieira, C., y J. Niño. 1994. El beneficio tributario debido al endeudamiento en las empresas chilenas. Estudios de Administración 1:31-41.

Martin, R., and T. Simin. 2003. Outlier-resistant estimates of beta. Finan. Analysts J. 59:56-69.

Moraleda, L., y L. Quezada. 2006. Decisiones financieras en Chile: teoría y práctica. $88 \mathrm{p}$. Tesis Ingeniero Comercial. Universidad de Talca, Talca, Chile.

Murray, L. 1995. An examination of beta estimation using daily Irish data. J. Bus. Finance Acc. 22:893-906.

Pereiro, L. 2001. The valuation of closely-held companies in Latin America. Emerg. Market Rev. 2:330-370.

RiskAmerica. 2006. Herramientas de ingeniería financiera para el mercado chileno. Disponible en http:// www.riskamerica.com Leído en enero de 2006.

Roll, R. 1977. A critique of the asset pricing theory's tests. J. Finan. Econ. 4:129-176.

Scholes, M., and J. Williams. 1977. Estimating betas for non-synchronous trading. J. Finan. Econ. 5:309-327.

Subtel. 2006. Subsecretaría de Telecomunicaciones (Subtel). Gobierno de Chile, Santiago, Chile. Disponible en http://procesostarifarios.subtel.cl Leído en enero de 2006.

Vasicek, O. 1973. A note on using cross-sectional information in Bayesian estimation of security betas. Amer. Econ. Rev. 28:1233-1239. 